\title{
COVID-19 en receptores de trasplante renal: ¿qué hemos aprendido tras 18 meses de pandemia?
}

\author{
Mario Fernández-Ruiz 1,2
}

${ }^{1}$ Unidad de Enfermedades Infecciosas. Hospital Universitario 12 de Octubre. Instituto de Investigación Hospital 12 de Octubre (i+12). Madrid. España

${ }^{2}$ Departamento de Medicina. Facultad de Medicina. Universidad Complutense. Madrid. España

Como citar este artículo: Fernández-Ruiz M. COVID-19 en receptores de trasplante renal: ¿qué hemos aprendido tras 18 meses de pandemia? Enferm Nefrol. 2021 Jul-Sep;24(3):219-31

\section{Resumen}

La infección por el SARS-CoV-2 (COVID-19) ha supuesto un importante impacto en la actividad trasplantadora en nuestro país. En su condición de paciente inmunodeprimido y con frecuentes comorbilidades, era esperable que la mortalidad y el riesgo de complicaciones asociadas a la COVID-19 en el receptor de trasplante renal (TR) fueran mayores en comparación con la población general, si bien la información al respecto en los primeros meses de la pandemia era muy limitada. Desde marzo de 2020 hemos mejorado rápidamente nuestro conocimiento acerca de la epidemiología, características clínicas y manejo de la COVID-19 post-trasplante. La presente revisión pretende recopilar la información disponible a julio de 2021 en respuesta a una serie de cuestiones relevantes: ¿cómo se manifiesta clínicamente la infección por SARS-CoV-2 en receptores de TR?, ¿cuáles son sus factores pronósticos?, ¿es más grave la COVID-19 en el contexto del TR respecto a los pacientes inmunocompetentes?, ¿de qué opciones de tratamiento antiviral disponemos actualmente para el receptor de TR?, ¿cuál es la experiencia disponible con los

\section{Correspondencia:}

Mario Fernández Ruiz

Email: mario_fdezruiz@yahoo.es tratamientos inmunomoduladores? y, por último, ¿son eficaces las vacunas frente a la COVID-19 basadas en ARN mensajero en esta población?. A pesar de los avances realizados, aún son varios los aspectos que debemos mejorar en nuestro abordaje de la infección por SARS-CoV-2 en el ámbito específico del TR.

PALABRAS CLAVE: COVID-19; SARS-CoV-2; trasplante renal; epidemiología; presentación clínica; pronóstico; tratamiento; vacunación.

\section{COVID-19 in kidney transplant recipients: what have we learned after 18 months of pandemic?}

\begin{abstract}
SARS-CoV-2 infection (COVID-19) has profoundly impacted transplant activity in our country. As immunocompromised hosts with common comorbidities, kidney transplant (KT) recipients were expected to have higher mortality and risk of complications associated to COVID-19 compared to the overall population. The available experience at the beginning of the pandemic, however, was very limited. Since March 2020 our
\end{abstract}


knowledge on the epidemiology, clinical features and management of post-transplant COVID-19 has rapidly evolved. The present review is aimed at summarizing the information generated by July 2021 to answer a number of relevant questions: How does SARS-CoV-2 infection present in KT recipients? What are the prognostic factors? Does COVID-19 entail a worse prognosis in the setting of KT as compared to non-immunocompromised individuals? What are the antiviral agents currently available for KT recipients? What is the experience with the use of immunomodulatory therapies? And lastly, are mRNA-based COVID-19 vaccines effective in this patient population? Despite notable advances achieved, we should still improve various aspects of our approach to SARS-CoV-2 infection in the specific setting of KT.

KEYWORDS: COVID-19; SARS-CoV-2; kidney transplantation; epidemiology; clinical features; outcome; treatment; vaccination.

\section{Introducción}

La enfermedad por coronavirus 2019 (COVID-19 por sus siglas en inglés) fue oficialmente declarada pandemia por la Organización Mundial de la Salud (OMS) el 11 de marzo de 2020. Desde entonces la infección por el coronavirus del síndrome respiratorio agudo grave 2 (SARS-CoV-2 por sus siglas en inglés) ha generado un notable impacto en todos los ámbitos sociales y asistenciales. El trasplante de órgano sólido (TOS) no podía constituir una excepción, con un descenso dramático en el número de procedimientos realizados en nuestro país a lo largo de los primeros meses de la pandemia․ La experiencia previa con otras infecciones por virus respiratorios, como la gripe, permitía anticipar que el receptor de trasplante renal (TR) -en su condición de paciente inmunodeprimido- estaría expuesto a un riesgo particularmente elevado de complicaciones derivadas de la COVID-19. No obstante, en aquellos primeros momentos las incertidumbres en torno a su presentación clínica, gravedad, tratamiento óptimo y prevención en esta población eran máximas. Cuando ya llevamos 18 meses luchando contra la COVID-19 estamos en condiciones de responder, al menos parcialmente, a muchas de estas cuestiones. Tal es el objetivo de la presente revisión.

\section{¿Cómo se manifiesta clínicamente la COVID-19 en el contexto del TR?}

Las manifestaciones de la infección por SARS-CoV-2 en receptores de TR no difieren sustancialmente de las observadas en la población general. Así, la fiebre es co- mún en la mayor parte de las series $(64,2 \%$ a $82,1 \%$ de los pacientes), seguida de la tos con o sin expectoración $(68,3 \%$ a $72,9 \%)$, disnea $(43,7 \%$ a $48,6 \%)$, cefalea $(30,6 \%)$, mialgias $(25 \%$ a $33,3 \%)$, rinorrea $(16,7 \%)$, y alteraciones del gusto y del olfato $(9,8 \%)^{2-8}$. Un aspecto hasta cierto punto diferencial en la presentación de la COVID-19 post-trasplante, es la elevada frecuencia de síntomas gastrointestinales (con una prevalencia comprendida entre el $11 \%$ y el $49 \%$ según los estudios $)^{3,5-7}$ y que, como se discute más adelante, parece conllevar implicaciones pronósticas ${ }^{9}$. Desde un punto de vista analítico destaca la presencia de linfopenia y la elevación de los reactantes de fase aguda ${ }^{2,4}$. El intervalo mediano entre el trasplante y el diagnóstico de la infección oscila entre 5 y 6 años 23,8,10,11. Es probable, no obstante, que las descripciones realizadas en los primeros meses de la pandemia estén sesgadas hacia las formas más graves que precisaron hospitalización y en las que se estableció el diagnóstico de COVID-19 mediante técnicas moleculares, como la reacción en cadena de la polimerasa con transcriptasa inversa (RT-PCR por sus siglas en inglés). La situación de colapso de los sistemas sanitarios vivida en muchos países occidentales implicó que un elevado número de casos leves o asintomáticos no llegaran a ser confirmados en aquel momento. En línea con esta hipótesis, un estudio reciente basado en el registro de la Organización Nacional de Trasplantes (ONT) mostró que el porcentaje de receptores de TOS con COVID-19 que precisaron ingreso a lo largo de 2020 fue superior en la primera ola respecto a la segunda ( $86,7 \%$ versus $58,1 \% ; \mathrm{P}<0,001)$, sugiriendo por tanto una mejoría de la capacidad diagnóstica y de notificación de $\operatorname{casos}^{12}$.

El riesgo de coinfección o de sobreinfección en el contexto de la COVID-19 post-trasplante es relativamente bajo y no parece superior al descrito para la población general ${ }^{13}$. En una amplia cohorte multicéntrica en EEUU con 482 receptores de TOS (dos tercios de los cuales habían recibido un TR), la incidencia acumulada de infección bacteriana a lo largo del seguimiento fue del $7,3 \%(8,0 \%$ en el grupo que precisó ingreso hospitalario), con Pseudomonas aeruginosa y Staphylococcus aureus como los microorganismos más frecuentes. Sólo tres pacientes $(0,6 \%)$ desarrollaron una infección fúngica invasora por hongo filamentoso (aspergilosis en dos de ellos), a los que habría que añadir un caso adicional de neumonía criptocócica ${ }^{11}$. Se han comunicado de forma anecdótica casos de aspergilosis pulmonar invasiva $^{14,15}$, mucormicosis ${ }^{16}$ y neumonía por Pneumocystis jirovecii ${ }^{17}$, complicaciones en las que habitualmente concurren otros factores de riesgo como el ingreso en 
la unidad de cuidados intensivos (UCI) con intubación prolongada o la administración de bolos de esteroides. De forma análoga a otras infecciones virales de tracto respiratorio como la gripe, el periodo de liberación del SARS-CoV-2 en la mucosa nasofaríngea es más prolongado en los receptores de TR que en los pacientes inmunocompetentes, y es frecuente la persistencia de la positividad de la RT-PCR durante al menos 3 semanas desde el inicio de los síntomas (con demostración incluso de viabilidad viral en el cultivo celular) ${ }^{18,19}$.

Una revisión sistemática con meta-análisis que incorporó datos procedentes de 74 estudios con 5.559 receptores de TR comunicó una mortalidad global del $23 \%$ y una incidencia de fracaso renal agudo (FRA) del $50 \%^{20}$. La mortalidad asociada a la COVID-19 en el contexto del TR, por otra parte, parece ser mayor que la observada en pacientes con enfermedad renal crónica (ERC) en hemodiálisis periódica ${ }^{21,22}$. Así lo sugiere, entre otros, un reciente estudio con 496 y 1.174 pacientes, respectivamente, en cada grupo. Si bien la mortalidad no ajustada a los 28 días fue menor en receptores de TR, el riesgo de muerte en el modelo ajustado fue un $78 \%$ mayor en comparación con los pacientes en hemodiálisis (hazard ratio [HR]: 1,78; intervalo de confianza del 95\% [IC 95\%]: 1,22-2,61) $)^{22}$. Esta mayor mortalidad asociada al TR respecto a la diálisis ha sido demostrada en otros trabajos $^{23}$. A pesar de que el manejo de la COVID-19 en sus formas graves conlleva la reducción o suspensión transitoria de la inmunosupresión (antiproliferativos en particular), el desarrollo de rechazo agudo del injerto durante las primeras semanas tras la infección ha sido anecdótico, con un solo caso entre los 482 receptores $(0,2 \%)$ incluidos en el citado estudio de Kates y colegas $^{11,24}$.

\section{¿Cuáles son los factores pronósticos en receptores de TR con COVID-19?}

Tras estos 18 meses de pandemia disponemos de varias cohortes multicéntricas en las que se ha analizado la evolución de la COVID-19 y sus factores pronósticos (tabla 1). En el registro multicéntrico coordinado por la Sociedad Española de Nefrología (SEN), que incluyó 1.011 receptores diagnosticados hasta diciembre de 2020, la mortalidad global fue del $21,7 \%$. EI análisis multivariante demostró que la presencia de neumonía (HR: 5,04; IC 95\%: 2,81-9,05) y la edad (HR por cada año: 1,06; IC 95\%: 1,05-1,08) actuaban como factores de mal pronóstico, en tanto que la presentación con síntomas gastrointestinales se asoció a una menor mortalidad (HR: 0,66; IC 95\%: 0,48$0,90)$. Uno de los hallazgos más relevantes fue que el desarrollo de infección por SARS-CoV-2 en los 6 primeros meses post-trasplante incrementaba de forma independiente el riesgo de muerte (HR: 1,64; IC 95\%: 1,07-2,50). De este modo los autores establecieron cuatro categorías pronósticas en función de la edad del receptor ( $<650 \geq 65$ años) y del intervalo transcurrido desde el trasplante ( $\leq 60>6$ meses). Los pacientes en el grupo 4 (de mayor edad y con trasplante más reciente) presentaban cuadros más graves (neumonía en el $81,4 \%$ de los casos) y una mortalidad del $54,5 \%$, que contrastaba con la de las restantes categorías: $34,5 \%$ en el grupo 3 ( $\geq 65$ años y $>6$ meses post-trasplante), $24,5 \%$ en el grupo $2(<65$ años y $\leq 6$ meses) y $11,3 \%$ en el grupo 1 ( $<65$ años y $\leq 6$ meses). Al igual que en el registro de la $0 \mathrm{NT}^{12}$, la mortalidad global fue menor en los pacientes diagnosticados durante la segunda ola (a partir de Julio de 2020), si bien esta diferencia se atenuaba cuando la comparación se centraba en las formas más graves que requirieron hospitalización o ingreso en UCI ${ }^{25}$. Partiendo igualmente del registro de la SEN se ha propuesto la existencia de distintos fenotipos clínicos con impacto pronóstico en la COVID-19 post-trasplante, ya que la presentación en forma de neumonía y disnea implica una mayor mortalidad respecto a los síntomas gastrointestinales aislados ${ }^{3}$. También se ha trabajado en esta línea en la población general, con el desarrollo y validación de un modelo de estratificación pronóstica basado en fenotipos clínicos ${ }^{26}$.

Un registro multinacional en el que participaron centros de Estados Unidos, Italia y España con 144 receptores de TR con precisaron ingreso comunicó una mortalidad aún más elevada (31,9\%). Una vez más la edad del receptor se asoció a un peor pronóstico (odds ratio [0R] por cada año: 1,07; IC 95\%: 1,02-1,14), así como la taquipnea, la disminución del filtrado glomerular estimado, y los niveles elevados de lactato deshidrogenasa (LDH) y de interleukina-6 (IL-6) en el momento del diagnóstico ${ }^{27}$. El análisis de otras cohortes multicéntricas también ha identificado la edad, la obesidad, la neumonía, la disnea, la linfopenia y la elevación de los niveles de LDH, IL-6 y otros reactantes de fase aguda (proteína $C$ reactiva $[P C R]$ o ferritina) como factores de mal pronóstico $0^{4,5,8,28}$. El desarrollo de FRA es una complicación frecuente en receptores de TR que precisan de ingreso, con una incidencia que oscila entre el $24 \%$ y el $83 \%$ según las series y la definición emplea$\mathrm{da}^{27,29,30}$. En un estudio reciente, menos de la mitad de los pacientes con esta complicación había recuperado por completo su función renal al cabo de 3 meses desde el diagnóstico de COVID-1929. 
Tabla 1. Resumen de las principales cohortes de receptores de TR con diagnóstico de COVID-19 publicadas hasta agosto de 2021.

\begin{tabular}{|c|c|c|c|c|}
\hline $\begin{array}{l}\text { Autor, país, } \\
\text { referencia }\end{array}$ & Tamaño muestral & $\begin{array}{l}\text { Periodo } \\
\text { de diagnóstico }\end{array}$ & $\begin{array}{l}\text { Mortalidad } \\
\text { (población, intervalo) }\end{array}$ & Factores pronósticos y principales hallazgos ${ }^{\mathrm{a}}$ \\
\hline $\begin{array}{l}\text { Favà et al. } \\
\text { España }^{8}\end{array}$ & 112 receptores de TR & $\begin{array}{l}4 \text { de marzo a } 17 \text { de } \\
\text { abril de } 2020\end{array}$ & $\begin{array}{l}26,9 \% \\
\text { (intrahospitalaria) }\end{array}$ & $\begin{array}{l}\text { Edad (HR [incremento unitario]: 1,103; IC 95\%: } \\
\text { 1,048-1,162) } \\
\text { SDRA en el día } 0 \text { (HR: } 2,091 \text {; IC 95\%: 1,031-8,233) } \\
\text { LDH (HR [incremento unitario]: } 1,003 ; \text { IC } \\
95 \%: 1,000-1,005 \text { ) }\end{array}$ \\
\hline Azzi et al. EEUU² & $\begin{array}{l}229 \text { receptores de TR } \\
\text { (incluye diagnóstico } \\
\text { serológico) }\end{array}$ & $\begin{array}{l}16 \text { de marzo a } 29 \\
\text { de julio de } 2020\end{array}$ & $\begin{array}{l}20,5 \% \text { (global, no se } \\
\text { especifica intervalo) }\end{array}$ & $\begin{array}{l}\text { Edad (OR [incremento de } 5 \text { años]: 1,48; IC 95\%: } \\
\text { 1,22-1,79) } \\
\text { Donante cadáver (0R: } 1,22 ; \text { IC } 95 \% \text { : } 1,067-1,74 \text { ) } \\
\text { Ausencia de vacunación frente a la gripe en la } \\
\text { temporada anterior (OR: } 1,13: \text { IC } 95 \%: 1,04-1,43 \text { ) } \\
\text { Niveles al diagnóstico de IL-6 ( } 47 \mathrm{vs.} 101 \mathrm{pg} / \mathrm{ml} ; \\
\mathrm{P}=0,036 \text { ) y pro-BNP ( } 1278 \mathrm{vs.} 2380 \mathrm{pg} / \mathrm{mL} ; \mathrm{P}= \\
0,031 \text { ) más altos en pacientes que fallecen respecto a } \\
\text { supervivientes }\end{array}$ \\
\hline $\begin{array}{l}\text { Mamode et al. } \\
\text { Reino Unido92 }\end{array}$ & 121 receptores de TR & $\begin{array}{l}\text { Marzo y abril de } \\
2020\end{array}$ & $\begin{array}{l}29,7 \% \\
\text { (intrahospitalaria) }\end{array}$ & Edad >60 años (0R: 4,3; IC 95\%: 1,8-10,2) \\
\hline Kute et al. India ${ }^{5}$ & 250 receptores de TR & $\begin{array}{l}23 \text { de marzo a } 15 \\
\text { de septiembre de } \\
2020\end{array}$ & $\begin{array}{l}11,6 \% \text { (global), } 14,5 \% \\
\text { (hospitalizados), } 47,2 \% \\
\text { (ingresados en UCI), } \\
96,7 \% \text { (VMI) }\end{array}$ & Creatinina sérica (HR: 5,424; IC 95\%: 1,294-2,273) \\
\hline Oto et al. Turquía ${ }^{4}$ & 109 receptores de TR & $\begin{array}{l}17 \text { de abril a } 1 \text { de } \\
\text { junio de } 2020\end{array}$ & $\begin{array}{l}12,8 \% \text { (no se especifica } \\
\text { intervalo) }\end{array}$ & $\begin{array}{l}\text { Cardiopatía isquémica (0R: 4,129; IC 95\%: } \\
\text { 1,104-15,442) } \\
\text { Creatinina sérica (0R: 1,681; IC 95\%: 1,083-2,608) }\end{array}$ \\
\hline $\begin{array}{l}\text { Villanego et al. } \\
\text { España }^{25}\end{array}$ & $\begin{array}{l}1011 \text { receptores de } \\
\text { TR }\end{array}$ & $\begin{array}{l}18 \text { de marzo a } 5 \text { de } \\
\text { diciembre de } 2020\end{array}$ & $\begin{array}{l}21,7 \% \text { (global), } 27,4 \% \\
\text { (primera ola), } 15,1 \% \\
\text { (segunda ola) }\end{array}$ & $\begin{array}{l}\text { Edad (HR [incremento unitario]: 1,06; IC 95\%: } \\
\text { 1,05-1,08) } \\
\text { Síntomas gastrointestinales (HR: 0,66; IC 95\%: } \\
\text { 0,48-0,90) } \\
\text { Tiempo desde el TR <6 meses (HR: 1,64: IC 95\%: } \\
\text { 1,07-2,50) } \\
\text { Neumonía (HR: 5,04; IC 95\%: 2,81-9,05) }\end{array}$ \\
\hline $\begin{array}{l}\text { Al Otaibi et al. } \\
\text { Kuwait }^{93}\end{array}$ & 104 receptores de TR & $\begin{array}{l}\text { Primera semana } \\
\text { de marzo a } 1 \text { de } \\
\text { agosto de } 2020\end{array}$ & $\begin{array}{l}10,6 \% \text { (global), } 35,5 \% \\
\text { (ingresados en UCI) }\end{array}$ & $\begin{array}{l}\text { Manejo de la inmunosupresión: } \\
\text { - mantenimiento sin cambios: } 45,2 \% \\
\text { - incremento de la dosis de esteroides: } 54,8 \% \\
\text { - suspensión de antiproliferativos y } \\
\text { anticalcineurínicos: } 10,6 \%\end{array}$ \\
\hline $\begin{array}{l}\text { Alshaqaq et al. } \\
\text { Arabia Saudi }^{94}\end{array}$ & 130 receptores de TR & $\begin{array}{l}1 \text { de marzo a } 31 \text { de } \\
\text { agosto de } 2020\end{array}$ & $10,8 \%$ (a los 28 días) & $\begin{array}{l}\text { Edad (HR [incremento unitario]: 1,06; IC 95\%: } \\
\text { 1,013-1,109) } \\
\text { Creatinina sérica (HR [incremento unitario]: 1,002; } \\
\text { IC 95\%: 1,00-1,004) } \\
\text { Tratamiento con azitromicina (HR: 6,380; IC 95\%: } \\
\text { 1,374-29,630) } \\
\text { FRA (HR: } 18,11 \text {; IC } 95 \%: 2,244-146,21 \text { ) }\end{array}$ \\
\hline $\begin{array}{l}\text { Caillard et al. } \\
\text { Francia95 }\end{array}$ & 494 receptores de TR & Primera ola & $20 \%$ (a los 60 días) & $\begin{array}{l}\text { Edad }>65 \text { años (HR: 7,33; IC 95\%: 1,91-28,1) } \\
\text { Procalcitonina >0,3 mg/L (HR: 3,73; IC 95\%: } \\
\text { 1,53-9,13) } \\
\text { Troponina T ultrasensible >20 ng/mL (HR: 2,91, IC } \\
\text { 95\%: } 1,02-8,34 \text { ) }\end{array}$ \\
\hline
\end{tabular}

BNP: péptido natriurético cerebral; FRA: fracaso renal agudo; HR: hazard ratio; IC 95\%; intervalo de confianza del 95\%; IL-6: interleuquina-6; LDH: lactato deshidrogenasa; 0R: odds ratio; SDRA: síndrome de distrés respiratorio del adulto; TR: trasplante renal; UCI: unidad de cuidados intensivos; VMI: ventilación mecánica invasiva.

a Sólo se indican los resultados de los análisis multivariantes para cada estudio. 


\section{¿Es más grave la COVID-19 en receptores de TR res- pecto a la población general?}

En vista de la elevada mortalidad global comunicada en las series recogidas durante la primera ola pandémica, varios estudios han tratado de esclarecer si la condición de receptor de TOS confiere en sí misma una peor evolución en la infección ${ }^{31}$. En ese sentido algunos autores han llegado a postular que, lejos de implicar un peor pronóstico, el tratamiento inmunosupresor post-trasplante podría reducir la intensidad de la fase hiperinflamatoria desencadenada por el SARSCoV-2 ${ }^{32}$, como vendría a corroborar el beneficio en términos de supervivencia o tiempo hasta la mejoría clínica demostrado en la población general con fármacos como la dexametasona ${ }^{33}$, los anticuerpos monoclonales dirigidos frente a la IL-6 o su receptor ${ }^{34}$, o el inhibidor de la Janus kinasa baricitinib ${ }^{35}$. En un estudio unicéntrico de casos y controles, Miarons y colegas compararon la evolución de la COVID-19 entre 46 receptores de TOS diagnosticados entre marzo y abril de 2020 y 116 pacientes no trasplantados emparejados por edad, género e índice de comorbilidad de Charlson. No detectaron diferencias significativas en la mortalidad a los 28 días $(37,0 \%$ versus $22,9 \%$, respectivamente; $\mathrm{P}=0,514)$, la necesidad de ingreso en UCI $(22,2 \%$ versus $18,4 \% ; P=0,161)$ o el tiempo hasta la defervescencia ( 7 frente a 9 días; $P=0,354$ ). Es destacable, en cambio, la tendencia hacia una menor incidencia de síndrome de distrés respiratorio del adulto (SDRA) en el grupo de TOS $(19,6 \%$ respecto al $27,1 \%$ de los controles no trasplantados; $P=0,060$ ), hallazgo que podría ser explicado por el potencial efecto protector de la inmunosupresión frente a la disfunción orgánica mediada por la respuesta inflamatoria ${ }^{36}$. Un estudio similar, aunque de naturaleza multicéntrica y con mayor tamaño muestral, realizado en Francia demostró que la frecuencia de COVID-19 grave (definida por la necesidad de ingreso en UCI o de ventilación mecánica invasiva [VMI] o el desarrollo de muerte) fue similar entre 306 receptores de TR y 795 controles no trasplantados $(43,8 \%$ versus $41,2 \%$, respectivamente; $P=0,21)$. Tras igualar ambos grupos por edad, índice de masa corporal y comorbilidades, la mortalidad a los 30 días fue mayor en receptores de TR $(17,9 \%$ versus $11,4 \%$; $\mathrm{P}=0,038$ ). Sin embargo, el trasplante no se mantuvo en el análisis multivariante como un factor independiente de mortalidad (a diferencia de la edad $>60$ años, la enfermedad cardiovascular, la presencia de disnea 0 de linfopenia, y el nivel elevado de PCR) ${ }^{37}$. Más recientemente un estudio realizado en EEUU que incluyó más de 2.000 receptores de TOS diagnosticados entre Enero y Septiembre de 2020 y 231.047 controles no trasplantados llegó a una conclusión similar: si bien la presencia de TOS se asociaba a una mayor mortalidad en el análisis crudo no ajustado (riesgo relativo [RR]: 2,47; IC 95\%: 2,05-2,96), esta asociación perdía su significación tras el ajuste por un score de propensión que tuvo en cuenta las diferencias basales entre ambos grupos en cuanto a comorbilidades y género (RR: 1,22; IC 95\%: 0,88-1,68). El grupo de TOS, no obstante, presentó una mayor incidencia de $\mathrm{FRA}^{38}$. Si bien otros autores tampoco han podido demostrar un impacto independiente del TOS sobre la evolución de la COVID-1939,40, en algún estudio el grupo de receptores presentó una mayor mortalidad y necesidad de VMI y de soporte con drogas vasoactivas en comparación con los pacientes no trasplantados apareados por edad ${ }^{41,42}$.

Aún a pesar de estas discrepancias, en conjunto estos resultados sugieren que la elevada mortalidad intrahospitalaria observada en pacientes con COVID-19 post-trasplante estaría condicionada en mayor medida por la edad del receptor, sus comorbilidades o el deterioro de la función renal que por el propio tratamiento inmunosupresor. Por tanto, la evidencia disponible no permite afirmar que la evolución de la infección por SARS-CoV-2 sea necesariamente peor entre receptores de TR en relación con la esperable en sujetos no inmunodeprimidos con edad y características clínicas similares.

\section{¿Qué opciones de tratamiento antiviral frente al SARS-CoV-2 están disponibles para el receptor de TR?}

Durante los primeros meses de la pandemia fue común el empleo de fármacos aprobados para otras indicaciones y que habían demostrado cierta actividad in vitro frente al SARS-CoV-2 u otros coronavirus, como el SARS-CoV o el del síndrome respiratorio de Oriente Medio (MERS-CoV por sus siglas en inglés) ${ }^{43}$. Así, los inhibidores de la proteasa (IP) como lopinavir o darunavir (potenciados con dosis bajas de ritonavir), la hidroxicloroquina, la azitromicina o el interferón- $\beta$ subcutáneo fueron ampliamente prescritos en receptores de TR con COVID-19, a pesar de la escasa (o nula) evidencia clínica disponible en ese momento y del riesgo de eventos adversos -incluyendo la potencial inducción de alorreactividad- y de interacciones farmacológicas. Por ejemplo, los IP actúan como potentes inhibidores de la isoforma 3A4 del citocromo P450 a través del cual se metabolizan los inhibidores de la calcineurina ${ }^{44}$. De este modo, y a pesar de la suspensión transitoria del tacrólimus seguida de una estrecha monitorización terapéutica en aquellos receptores en los que se 
iniciaba tratamiento con un IP, la presencia de niveles plasmáticos excesivos fue habitual y se acompañó en ocasiones de toxicidad y eventos clínicos desfavorables, incluyendo FRA y necesidad de diálisis ${ }^{45,46}$. El registro de la ONT revela que hasta el $86 \%$ de los receptores de TR con diagnóstico de COVID-19 hasta Julio de 2020 fue tratado con hidroxicloroquina, el $37 \%$ recibió un IP (habitualmente lopinavir/ritonavir), el 57\% azitromicina, y el $5 \%$ interferón- $\beta^{47}$. Por desgracia, ninguno de estos fármacos ha demostrado beneficio clínico alguno (tiempo hasta la mejoría clínica, necesidad de VMI o mortalidad) en la práctica totalidad de los ensayos clínicos (EC) publicados a partir de mediados de 2020 y con independencia de la gravedad de la infección, si bien ninguno de ellos estaba específicamente centrado en la población trasplantada ${ }^{48-52}$. Como consecuencia, su prescripción en receptores de TR decayó de forma notable a partir de la segunda ola hasta su práctica desaparición en el momento actual ${ }^{12}$.

El remdesivir es un análogo de la adenosina que inhibe de forma competitiva la ARN polimerasa viral. Al ser incorporado como un falso nucleósido a la cadena de ARN induce la terminación prematura de su proceso de síntesis. Presenta actividad in vitro frente a un amplio espectro de virus ARN, como el virus Ébola, el virus Marburg o el MERS-CoV ${ }^{53}$. En el EC ACTT-1 el tratamiento con remdesivir disminuyó respecto al placebo el tiempo hasta la mejoría clínica en pacientes con COVID-19 que requerían oxigenoterapia (media de 10 días frente a 15 días, respectivamente; $P<0,001$ ), así como la necesidad de VMI (sin alcanzar en este caso la significación estadística). No se detectó, sin embargo, efecto sobre la mortalidad. Cabe destacar que el beneficio asociado al remdesivir no fue extensivo a pacientes sin insuficiencia respiratoria 0 , en el extremo opuesto de gravedad, a aquellos que ya estaban recibiendo ventilación mecánica ${ }^{54}$. Por otra parte, el EC SOLIDARITY auspiciado por la OMS no reveló ningún beneficio en términos de mortalidad o duración del ingreso hospitalario ${ }^{48}$. Tampoco se ha podido demostrar que el uso de remdesivir acelere el aclaramiento viral o reduzca los parámetros inflamatorios en comparación con el tratamiento habitual ${ }^{55}$.

El empleo de remdesivir en ámbito del TR ha estado limitado por la ausencia de datos específicos acerca de su eficacia y seguridad y por los criterios de prescripción establecidos por las autoridades regulatorias en España. Así, sólo fue administrado en el $4,8 \%$ de los 861 receptores de TOS incluidos en el registro de la ONT durante la segunda ola ${ }^{12}$. Por otra parte, está contra- indicado en presencia de un aclaramiento de creatinina menor de $30 \mathrm{~mL} / \mathrm{min}$ debido a la potencial acumulación del excipiente (sulfobutil-éter- $\beta$-ciclodextrina sódica), lo cual limita su empleo en caso de mala función del injerto renal. Un análisis retrospectivo del registro de la SEN ha comunicado recientemente la experiencia con el uso de remdesivir en 51 receptores de TR, la mayor parte de los cuales $(54,9 \%)$ precisaban ventilación mecánica. EI remdesivir fue iniciado al cabo de una mediana de 2 días desde el ingreso. La mortalidad global fue del $18,9 \%$, y el $11,7 \%$ de los receptores desarrolló un FRA potencialmente atribuible al fármaco. Hubo dos episodios de toxicidad hepática de grado 1 . No obstante, en ninguno caso fue necesaria la discontinuación precoz debido a la aparición de eventos adversos, y todos los pacientes con FRA que sobrevivieron recuperaron la función del injerto. El carácter observacional del estudio y la ausencia de grupo control, por desgracia, limitan la interpretación de estos resultados ${ }^{56}$.

Un abordaje prometedor consiste en el empleo de anticuerpos monoclonales neutralizantes dirigidos frente a la región de unión al receptor de la glucoproteína de la espícula del SARS-CoV-2, impidiendo la entrada del virus en la célula. La administración de casirivimab e imdevimab en una infusión intravenosa única fue bien tolerada y redujo la carga viral en el frotis nasofaríngeo en pacientes ambulatorios ${ }^{57}$. Más recientemente, un EC controlado con placebo demostró que el tratamiento con bamlanivimab y etesevimab durante los tres primeros días desde el diagnóstico en pacientes no hospitalizados con COVID-19 leve o moderada y factores de riesgo de progresión reducía la necesidad de ingreso $(2,1 \%$ en el grupo experimental frente a $7,0 \%$ en el de placebo; reducción absoluta de riesgo: $-4,8$ puntos porcentuales; $\mathrm{P}<0,001$ ) y la mortalidad. Entre los criterios de inclusión figuraba el tratamiento inmunosupresor previo, si bien no se aportan detalles al respecto $0^{58}$. La experiencia con el uso de estas combinaciones de anticuerpos en receptores de TOS es hasta el momento escasa. En una serie de 25 pacientes (incluyendo 17 receptores de TR) con COVID-19 leve o moderada, ninguno de los participantes presentó progresión de los síntomas o requerimiento de oxigenoterapia tras la administración de casirivimab-imdevimab o bamlanivimab ${ }^{59}$. Además de la precocidad del tratamiento, la necesidad de infusión intravenosa constituye una dificultad logística añadida, por lo que se podría reservar el uso de estos anticuerpos en casos seleccionados de receptores de TR con pocos días de evolución de los síntomas, o incluso de forma preventiva tras un contacto de riesgo en sujetos no vacunados. 


\section{¿Cuál es la experiencia disponible con los tratamien- tos inmunomoduladores en receptores de TR con COVID-19?}

Una vez demostrado que gran parte del daño multiorgánico que complica el curso de las formas graves de COVID-19 (SDRA, coagulopatía, fenómenos trombóticos, etc) está mediado por una reacción inflamatoria excesiva frente al SARS-CoV-2 desencadenada por la inmunidad innata del huésped ${ }^{60}$, se comenzó a postular ya desde las primeras semanas de la pandemia el empleo de diversos abordajes inmunomoduladores. Por analogía con escenarios como el de la sepsis o el tratamiento con linfocitos T con receptor de antígeno quimérico (CAR-T por sus siglas en inglés), se ha generalizado el término de síndrome o "tormenta" de liberación de citoquinas para hacer referencia a este proceso $0^{61}$. El papel fundamental desempeñado por la citoquina proinflamatoria y pleiotrópica IL-6 dio pie a priorizar el uso de fármacos capaces de bloquear sus acciones biológicas, como el tocilizumab o el sarilumab. Si bien los EC iniciales en pacientes no inmunodeprimidos proporcionaron resultados negativos o discordantes en cuanto a la eficacia del tocilizumab ${ }^{62}$, los estudios más recientes con un elevado tamaño muestral y periodos de seguimiento más prolongados han permitido demostrar un beneficio significativo - aunque discreto en términos absolutos - en el riesgo de muerte 0 de progresión a VMI ${ }^{63-65}$.

La evidencia disponible en la población trasplantada, por desgracia, es mucho más limitada al no haberse realizado hasta la fecha ningún estudio de intervención ${ }^{66}$. Por ese motivo, el uso de tocilizumab en el ámbito del TR es fruto fundamentalmente de la extrapolación de los EC realizados en pacientes no trasplantados y de pequeñas series de casos sin grupo control67,68. En el registro de la ONT, por ejemplo, el $21 \%$ de los receptores de TR fueron tratados con este anticuerpo monoclonal durante la primera ola ${ }^{47}$. La experiencia multicéntrica en nuestro país con 80 receptores de TR en los que el tocilizumab fue administrado al cabo de una mediana de 5 días desde el ingreso reveló una elevada mortalidad (32,5\%), particularmente condicionada por la edad del paciente. Los niveles séricos de PCR disminuyeron tras el tratamiento (con descensos mayores en supervivientes) y su variación se correlacionó con la mortalidad (HR por cada $\mathrm{mg} / \mathrm{L}: 1,01$; IC $95 \%$ : 1,00-1,02). Aunque el tratamiento con tocilizumab fue seguro en términos globales, hubo 6 casos de neumonía bacteriana en pacientes también tratados con esteroides ${ }^{69}$. El tratamiento con tocilizumab en el contexto de la COVID-19 post-trasplante se acompaña del incremento de los niveles séricos de IL-6 -hallazgo ya descrito en pacientes inmunocompe- tentes y que resulta de la saturación del receptor de la citoquina ${ }^{70}$ - y del receptor soluble de la IL-2 (sIL2R), así como de la reducción de la IL-1071. Una de las pocas experiencias comparativas es la de Pereira y colegas, que llevaron a cabo un estudio de casos y controles con 29 receptores de TOS (TR en 15 de ellos) tratados con tocilizumab y otros 29 controles emparejados por edad, hipertensión, presencia de ERC y tratamiento con dosis altas de esteroides durante el episodio de COVID-19. Los autores no observaron diferencias entre los pacientes tratados o no con tocilizumab en términos de mortalidad ( $41 \%$ versus $28 \%$, respectivamente; $P=0,27$ ) 0 infección secundaria ( $34 \%$ versus $24 \% ; P=0,55)^{72}$. En una reducida cohorte de 33 receptores de TR -de los que 19 fueron tratados con algún agente dirigido frente a la IL-6 (tocilizumab) o IL-1 (anakinra)- tampoco se pudo demostrar un beneficio significativo en cuanto a la necesidad de ingreso en UCI o mortalidad ${ }^{73}$. Como consecuencia tal vez del carácter no concluyente de esta limitada evidencia, el empleo de tocilizumab parece haber disminuido de forma significativa en nuestro país a partir de la segunda ola ${ }^{12}$.

Por otra parte, el EC RECOVERY demostró que el tratamiento con dexametasona $(6 \mathrm{mg}$ diarios durante 10 días) reducía la mortalidad a los 28 días en pacientes con COVID-19 y necesidad de oxigenoterapia ${ }^{33}$. Estos resultados y los de otros estudios ${ }^{74}$ han consolidado el uso con corticosteroides a dosis bajas-intermedias en el abordaje estándar de la neumonía por SARS-CoV-2 con insuficiencia respiratoria. Este beneficio se ha extrapolado al contexto del TR, si bien una vez más carecemos de EC específicamente diseñados para esta población. Por tanto, la práctica más común en el registro de la ONT a lo largo de las dos primeras olas en relación con el tratamiento inmunosupresor basal en receptores de TOS con necesidad de ingreso consistió en la suspensión del antiproliferativo (micofenolato mofetilo o sódico y azatioprina) y en el aumento de dosis del corticosteroide o su reintroducción ${ }^{12,47}$. No obstante, el manejo óptimo de la inmunosupresión en la COVID-19 post-trasplante sigue sin estar bien establecido. Un meta-análisis basado en 202 receptores de TOS, de hecho, recomienda el mantenimiento de la misma sin necesidad de ajuste y sugiere un papel protector para el tacrólimus, si bien los propios autores reconocen la limitada calidad de los datos disponibles ${ }^{75}$.

\section{¿Es eficaz la vacunación frente a la COVID-19 en re- ceptores de TR?}

Las vacunas basadas en la tecnología de ARN mensajero (que codifica la secuencia completa de la gluco- 
proteína de la espícula del SARS-CoV-2) han mostrado una efectividad clínica en torno al $95 \%$ a la hora de prevenir el desarrollo de COVID-19 en la población general, tanto en el contexto de $\mathrm{EC}^{76,77}$ como en estudios en vida real ${ }^{76}$. Por desgracia, un número creciente de estudios revela que la eficacia de cualquiera de estas vacunas (BNT162b2 y mRNA-1273) es sustancialmente menor en receptores de TR. Las tasas de seroconversión oscilan entre el $26,5 \%{ }^{78}$ y el $47,8 \%{ }^{79}$ cuando se analiza el desarrollo de anticuerpos IgG totales frente a la espícula del SARS-CoV-2 mediante ELISA, CLIA o Luminex. Aunque la información disponible es más limitada, la generación post-vacunal de una respuesta específica mediada por células también parece estar comprometida, con una tasa del $54,7 \%$ en un estudio reciente que empleó una técnica de ELISpot $^{78}$. Como consecuencia de ello, se ha descrito el desarrollo de COVID-19 en receptores que habían recibido la pauta completa de vacunación, incluyendo casos graves que precisaron de ingreso y con desenlace fatal ${ }^{80,81}$. La administración de mofetil micofenolato dentro del régimen inmunosupresor de mantenimiento ha sido identificada como un factor que disminuye las posibilidades de seroconversión tras la vacunación ${ }^{79,82}$, tal y como ya había sido observado para la vacuna frente a la gripe ${ }^{83}$. Otros factores asociados a la ausencia de respuesta mediada por anticuerpos en diversos estudios incluyen la edad del receptor, la presencia de linfopenia, el tiempo transcurrido desde el trasplante, los niveles plasmáticos de fármacos anticalcineurínicos y ciertas comorbilidades como la diabetes ${ }^{78,82,84,85}$. Se ha comprobado igualmente que el tratamiento con belatacept (un inhibidor de la co-estimulación linfocitaria) ejerce un impacto especialmente deletéreo sobre el desarrollo de respuesta vacunal, tanto humoral como celular ${ }^{86,87}$. Por otra parte, y de forma análoga a la población no trasplantada, la administración de una única dosis de vacuna induce una respuesta humoral robusta en presencia de inmunidad natural preexistente en receptores que ha presentado previamente COVID-1988. En términos globales, las vacunas basadas en ARN mensajero presentan un perfil de seguridad en receptores de TR similar al observado en la población general, sin que se haya comunicado el desarrollo de anticuerpos donante-específicos de novo o rechazo del injerto ${ }^{89}$. En vista de la menor eficacia de la vacunación en población trasplantada se están diseñando estrategias alternativas que permitan incrementar la tasa de seroconversión entre las que destaca la administración de una tercera dosis de BNT162b2 o mRNA-1273, con resultados preliminares esperanzadores ${ }^{90,91}$.

\section{Conclusiones y cuestiones para el futuro}

Como ponen de manifiesto las líneas precedentes, nuestro conocimiento acerca de la epidemiología, presentación clínica y abordaje terapéutico de la COVID-19 en el ámbito del TR ha evolucionado rápidamente en apenas una año y medio, redundando en un mejor pronóstico para nuestros pacientes. Por desgracia, seguimos careciendo de agentes antivirales realmente eficaces frente al SARS-CoV-2. Mejorar la inmunogenicidad subóptima de las vacunas basadas en ARN mensajero en receptores de TOS constituye igualmente un desafío de cara al futuro. La seguridad del TR en candidatos convalecientes de COVID-19 o la utilización de órganos abdominales procedentes de donantes con infección pasada (o incluso activa) serán sin duda objeto de investigación a lo largo de los próximos meses. Mientras tanto, deberemos mantener el nivel de recomendación hacia nuestros pacientes receptores de TR en relación con las medidas de prevención (uso de mascarilla, distancia social, higiene de manos, etc) como la mejor medida para minimizar los riesgos asociados a esta infección.

Recepción: 20-07-21

Aceptación: 10-08-21

Publicación: 30-09-21

\section{Bibliografía}

1. Domínguez-Gil B, Coll E, Fernández-Ruiz M, Del Rio F, Zaragoza R, Rubio JJ, et al. CoVID-19 in Spain: Transplantation in the midst of the pandemic. Am J Transplant. 2020;20(9):2593-8.

2. Azzi Y, Parides M, Alani 0, Loarte-Campos P, Bastarsh $\mathrm{R}$, Forest $\mathrm{S}$, et al. COVID-19 infection in kidney transplant recipients at the epicenter of pandemics. Kidney Int. 2020;98(6):1559-67.

3. Crespo M, Mazuecos A, Rodrigo E, Gavela E, Villanego F, Sánchez-Álvarez E, et al. Respiratory and Gastrointestinal COVID-19 Phenotypes in Kidney Transplant Recipients. Transplantation. 2020;104(11):2225-33.

4. Oto OA, Ozturk S, Turgutalp K, Arici M, Alpay N, Merhametsiz 0 , et al. Predicting the outcome of $\mathrm{CO}$ VID-19 infection in kidney transplant recipients. BMC Nephrol. 2021;22(1):100. 
5. Kute VB, Bhalla AK, Guleria S, Ray DS, Bahadur M M, Shingare $A$, et al. Clinical Profile and Outcome of COVID-19 in 250 Kidney Transplant Recipients: A Multicenter Cohort Study From India. Transplantation. 2021;105(4):851-60.

6. Pierrotti LC, Reusing Junior J0, Freire MP, Barros-Machado DJ, Megale-Moreira R, Ventura CG, et al. COVID-19 among kidney-transplant recipients requiring hospitalization: preliminary data and outcomes from a single-center in Brazil. Transpl Int. 2020;33(12):1837-42.

7. Caillard S, Anglicheau D, Matignon M, Durrbach $A$, Greze C, Frimat $L$, et al. An initial report from the French SOT COVID Registry suggests high mortality due to COVID-19 in recipients of kidney transplants. Kidney Int. 2020;98(6):1549-58.

8. Fava A, Cucchiari D, Montero N, Toapanta N, Centellas FJ, Vila-Santandreu A, et al. Clinical characteristics and risk factors for severe COVID-19 in hospitalized kidney transplant recipients: A multicentric cohort study. Am J Transplant. 2020;20(11):3030-41.

9. Guillén E, Pineiro GJ, Revuelta I, Rodríguez D, Bodro $M$, Moreno $A$, et al. Case report of COVID-19 in a kidney transplant recipient: Does immunosuppression alter the clinical presentation? Am J Transplant. 2020;20(7):1875-8.

10. Elías M, Pievani D, Randoux C, Louis K, Denis B, Delion $A$, et al. COVID-19 Infection in Kidney Transplant Recipients: Disease Incidence and Clinical Outcomes. J Am Soc Nephrol. 2020;31(10):2413-23.

11. Kates OS, Haydel BM, Florman SS, Rana M M, Chaudhry ZS, Ramesh MS, et al. COVID-19 in solid organ transplant: A multi-center cohort study. Clin Infect Dis. 2020. [En prensa]. DOI: https://doi.org/10.1093/ $\mathrm{cid} / \mathrm{ciaal} 097$.

12. Coll $E$, Fernández-Ruiz $M$, Padilla $M$, Moreso $F$, Hernández-Vicente A, Yañez I, et al. COVID-19 in Solid Organ Transplant Recipients in Spain Throughout 2020: Catching the Wave? Transplantation. 2021. [En prensa]. D0I: https://doi.org/10.1097/ TP.0000000000003873.

13. García-Vidal C, Sanjuan G, Moreno-García E, Puerta-Alcalde P, Garcia-Pouton N, Chumbita M, et al. Incidence of co-infections and superinfections in hos- pitalized patients with COVID-19: a retrospective cohort study. Clin Microbiol Infect. 2021;27(1):83-8.

14. Trujillo $H$, Fernández-Ruiz $M$, Gutiérrez $E$, Sevillano $A$, Caravaca-Fontán $F$, Morales $E$, et al. Invasive pulmonary aspergillosis associated with COVID-19 in a kidney transplant recipient. Transpl Infect Dis. 2021;23(2):e13501.

15. Cruzado Vega LL, Santos García A. SARS-CoV-2 and Aspergillus pneumonia in kidney transplantation: More frequent than we think? Nefrologia (Engl Ed). 2021.

16. Arana C, Cuevas Ramírez RE, Xipell M, Casals J, Moreno A, Herrera $S$, et al. Mucormycosis associated with COVID-19 in two kidney transplant patients. Transpl Infect Dis. 2021:e13652.

17. Skonieczny $P$, Heleniak Z, Szostakiewicz M, Kuziemski K, Debska-Slizien A. Coinfection of COVID-19 and pneumocystosis in a patient after kidney transplantation. Pol Arch Intern Med. $2021 ; 131(6): 566-7$.

18. Benotmane I, Risch S, Doderer-Lang C, Caillard S, Fafi-Kremer S. Long-term shedding of viable SARSCoV-2 in kidney transplant recipients with COVID-19. Am J Transplant. 2021.

19. Italiano J, Bush R, Acharya R, Upadhyay K. Persistent viral shedding despite seroconversion in a kidney transplant recipient with severe extrapulmonary $\mathrm{CO}$ VID-19. BMJ Case Rep. 2020;13(11).

20.20. Kremer $D$, Pieters $T T$, Verhaar $M C$, Berger $S P$, Bakker SJL, van Zuilen AD, et al. A Systematic Review and Meta-analysis of COVID-19 in Kidney Transplant Recipients: Lessons to be Learned. Am J Transplant. 2021. [En prensa].

21. Jager KJ, Kramer A, Chesnaye NC, Couchoud C, Sánchez-Álvarez E, Garneata $L$, et al. Results from the ERA-EDTA Registry indicate a high mortality due to COVID-19 in dialysis patients and kidney transplant recipients across Europe. Kidney Int. 2020;98(6):1540-8.

22. Goffin $E$, Candellier A, Vart $P$, Noordzij M, Arnol M, Covic $A$, et al. COVID-19 related mortality in kidney transplant and hemodialysis patients: a comparative, prospective registry based study. Nephrol Dial Transplant. 2021. 
23. Clarke C, Lucisano G, Prendecki M, Gleeson S, Martin $\mathrm{P}$, Ali M, et al. Informing the Risk of Kidney Transplantation Versus Remaining on the Waitlist in the Coronavirus Disease 2019 Era. Kidney Int Rep. 2021;6(1):46-55.

24. Mahalingasivam $V$, Craik $A$, Tomlinson $L A$, Ge $L$, Hou k, Wang $Q$, et al. A Systematic Review of COVID-19 and Kidney Transplantation. Kidney Int Rep. 2021;6(1):24-45.

25. Villanego $F$, Mazuecos A, Pérez-Flores IM, Moreso $F$, Andrés $A$, Jiménez-Martín $C$, et al. Predictors of severe COVID-19 in kidney transplant recipients in the different epidemic waves: Analysis of the Spanish Registry. Am J Transplant. 2021;21(7):2573-82.

26. Gutiérrez-Gutiérrez $B$, Del Toro MD, Borobia AM, Carcas A, Jarrín I, Yllescas $M$, et al. Identification and validation of clinical phenotypes with prognostic implications in patients admitted to hospital with COVID-19: a multicentre cohort study. Lancet Infect Dis. 2021;21(6):783-92.

27. Cravedi P, Mothi SS, Azzi Y, Haverly M, Farouk SS, Pérez-Sáez MJ, et al. COVID-19 and kidney transplantation: Results from the TANGO International Transplant Consortium. Am J Transplant. 2020;20(11):3140-8.

28. Salto-Alejandre $S$, Jiménez-Jorge $S$, Sabe N, Ramos-Martínez A, Linares $L$, Valerio $M$, et al. Risk factors for unfavorable outcome and impact of early post-transplant infection in solid organ recipients with COVID-19: A prospective multicenter cohort study. PLoS One. 2021;16(4):e0250796.

29. Bajpai D, Deb S, Bose S, Gandhi C, Modi T, Katyal A, et al. Recovery of kidney function after AKI because of COVID-19 in kidney transplant recipients. Transpl Int. 2021;34(6):1074-82.

30. Elec $A D$, Oltean $M$, Goldis $P$, Cismaru $C$, Lupse $M$, Muntean A, et al. COVID-19 after kidney transplantation: Early outcomes and renal function following antiviral treatment. Int J Infect Dis. 2021;104:426-32.

31. Van Delden C. Is transplantation per se a risk factor for worse outcome of SARS-CoV-2 infection in kidney transplant recipients? Transpl Int. 2021;34(5):775-7.

32. Romanelli A, Mascolo S. Immunosuppression drug-related and clinical manifestation of Coronavirus disea- se 2019: A therapeutical hypothesis. Am J Transplant. 2020;20(7):1947-8.

33. Group RC, Horby P, Lim WS, Emberson JR, Mafham $\mathrm{M}$, Bell $\mathrm{JL}$, et al. Dexamethasone in Hospitalized Patients with COVID-19. N Engl J Med. 2021;384(8):693-704.

34. The WHO Rapid Evidence Appraisal for COVID-19 Therapies (REACT) Working Group. Association Between Administration of IL-6 Antagonists and Mortality Among Patients Hospitalized for COVID-19: A Meta-analysis. JAMA. 2021;326(6):499-518.

35. Kalil AC, Patterson TF, Mehta AK, Tomashek KM, Wolfe CR, Chazaryan V, et al. Baricitinib plus Remdesivir for Hospitalized Adults with COVID-19. N Engl J Med. 2021;384(9):795-807.

36. Miarons M, Larrosa-García M, García-García $S$, Los-Arcos I, Moreso F, Berastegui C, et al. COVID-19 in Solid Organ Transplantation: A Matched Retrospective Cohort Study and Evaluation of Immunosuppression Management. Transplantation. 2021;105(1):138-50.

37. Caillard S, Chavarot $N$, Francois $H$, Matignon $M$, Greze C, Kamar N, et al. Is COVID-19 infection more severe in kidney transplant recipients? Am J Transplant. 2021;21(3):1295-303.

38. Hadi YB, Naqvi SFZ, Kupec JT, Sofka S, Sarwari A. Outcomes of COVID-19 in Solid Organ Transplant Recipients: A Propensity-matched Analysis of a Large Research Network. Transplantation. 2021;105(6):1365-71.

39. Chavarot N, Gueguen J, Bonnet G, Jdidou M, TrimaiIle A, Burger C, et al. CoVID-19 severity in kidney transplant recipients is similar to nontransplant patients with similar comorbidities. Am J Transplant. 2021;21(3):1285-94.

40. Sharma $P$, Chen V, Fung CM, Troost JP, Patel VN, Combs M, et al. COVID-19 Outcomes Among Solid Organ Transplant Recipients: A Case-control Study. Transplantation. 2021;105(1):128-37.

41. Fisher AM, Schlauch D, Mulloy M, Dao A, Reyad AI, Correl M, et al. Outcomes of COVID-19 in hospitalized solid organ transplant recipients compared to a matched cohort of non-transplant patients at a natio- 
nal healthcare system in the United States. Clin Transplant. 2021;35(4):e14216.

42. Nair V, Jandovitz N, Hirsch JS, Abate M, Satapathy SK, Roth N, Miyara SJ, et al. An early experience on the effect of solid organ transplant status on hospitalized COVID-19 patients. Am J Transplant. 2021;21(7):2522-31.

43. Pawar AY. Combating devastating COVID-19 by drug repurposing. Int $\mathrm{J}$ Antimicrob Agents. 2020;56(2):105984.

44. Vogel M, Voigt $E$, Michaelis HC, Sudhop T, Wolff M, Türler $A$, et al. Management of drug-to-drug interactions between cyclosporine $A$ and the protease-inhibitor lopinavir/ritonavir in liver-transplanted HIV-infected patients. Liver Transpl. 2004;10(7):939-44.

45. Fernández-Ruiz $M$, Andres $A$, Loinaz $C$, Delgado JF, López-Medrano F, San Juan R, et al. COVID-19 in solid organ transplant recipients: A single-center case series from Spain. Am J Transplant. 2020;20(7):1849-58.

46. Xia T, Wang Y. Coronavirus disease 2019 and transplantation: The combination of lopinavir/ritonavir and hydroxychloroquine is responsible for excessive tacrolimus trough level and unfavorable outcome. Am J Transplant. 2020;20(9):2630-1.

47. Coll $E$, Fernández-Ruiz $M$, Sánchez-Álvarez JE, Martínez-Fernández JR, Crespo M, Gayoso J, et al. COVID-19 in transplant recipients: The Spanish experience. Am J Transplant. 2021;21(5):1825-37.

48. Consortium WHOST, Pan $\mathrm{H}$, Peto $\mathrm{R}$, Henao-Restrepo AM, Preziosi MP, Sathiyamoorthy $V$, et al. Repurposed Antiviral Drugs for COVID-19 - Interim WHO Solidarity Trial Results. N Engl J Med. 2021;384(6):497-511.

49. Group RC. Azithromycin in patients admitted to hospital with COVID-19 (RECOVERY): a randomised, controlled, open-label, platform trial. Lancet. 2021;397(10274):605-12.

50. Group RC. Lopinavir-ritonavir in patients admitted to hospital with COVID-19 (RECOVERY): a randomised, controlled, open-label, platform trial. Lancet. 2020.

51. Group RC, Horby P, Mafham M, Linsell L, Bell JL, Staplin N, et al. Effect of Hydroxychloroquine in Hos- pitalized Patients with COVID-19. N Engl J Med. 2020;383(21):2030-40.

52. Oldenburg CE, Pinsky BA, Brogdon J, Chen C, Ruder $\mathrm{K}$, Zhong $\mathrm{L}$, et al. Effect of Oral Azithromycin vs Placebo on COVID-19 Symptoms in Outpatients With SARS-CoV-2 Infection: A Randomized Clinical Trial. JAMA. 2021.

53. Jorgensen SCJ, Kebriaei R, Dresser LD. Remdesivir: Review of Pharmacology, Pre-clinical Data, and Emerging Clinical Experience for COVID-19. Pharmacotherapy. 2020;40(7):659-71.

54. Beigel JH, Tomashek KM, Dodd LE, Mehta A, Zingman BS, Kalil AC, et al. Remdesivir for the Treatment of COVID-19 - Final Report. N Engl J Med. 2020;383(19):1813-26.

55. Barratt-Due A, Olsen IC, Nezvalova-Henriksen K, Kåsine $T$, Lund-Johansen $F$, Hoel $H$, et al. Evaluation of the Effects of Remdesivir and Hydroxychloroquine on Viral Clearance in COVID-19: A Randomized Trial. Ann Intern Med. 2021.

56. Buxeda A, Arias-Cabrales C, Pérez-Sáez MJ, Pascual $\mathrm{J}$, Crespo M. Use and safety of remdesivir in kidney transplant recipients with COVID-19. Kidney Int Rep. 2021.

57. Weinreich DM, Sivapalasingam S, Norton $T$, Ali $S$, Gao J, Bhore R, et al. REGN-COV2, a Neutralizing Antibody Cocktail, in Outpatients with COVID-19. N Engl J Med. 2021;384(3):238-51.

58. Dougan M, Nirula A, Azizad M, Mocherla B, Gottlieb $\mathrm{RL}$, Chen $\mathrm{P}$, et al. Bamlanivimab plus Etesevimab in Mild or Moderate COVID-19. N Engl J Med. 2021.

59. Dhand A, Lobo SA, Wolfe K, Feola N, Lee L, Nog R, et al. Casirivimab-imdevimab for Treatment of $\mathrm{CO}$ VID-19 in Solid Organ Transplant Recipients: An Early Experience. Transplantation. 2021;105(7):e68-e9.

60. McGonagle D, Sharif K, O'Regan A, Bridgewood C. The Role of Cytokines including Interleukin- 6 in COVID-19 induced Pneumonia and Macrophage Activation Syndrome-Like Disease. Autoimmun Rev. 2020;19(6):102537.

61. Fajgenbaum DC, June CH. Cytokine Storm. N Engl J Med. 2020;383(23):2255-73. 
62. Fernández-Ruiz $M$, López-Medrano $F$, Aguado JM. Tocilizumab for the treatment of COVID-19. Expert Opin Biol Ther. 2021;21(4):431-4.

63. RECOVERY Collaborative Group. Tocilizumab in patients admitted to hospital with COVID-19 (RECOVERY): a randomised, controlled, open-label, platform trial. Lancet. 2021;397(10285):1637-45.

64. REMAP-CAP Investigators, Gordon AC, Mouncey $\mathrm{PR}$, et al. Interleukin-6 Receptor Antagonists in Critically Ill Patients with COVID-19. N Engl J Med. 2021;384(16):1491-502.

65. Mariette $X$, Hermine 0 , Tharaux $P L$, Resche-Rigon $M$, Steg PG, Porcher R, et al. Effectiveness of Tocilizumab in Patients Hospitalized With COVID-19: A Follow-up of the CORIMUNO-TOCI-1 Randomized Clinical Trial. JAMA Intern Med. 2021.

66. Fernández-Ruiz M, Aguado JM. Immunomodulatory Therapies for COVID-19 in Solid Organ Transplant Recipients. Curr Transplant Rep. 2020:1-11.

67. Guella A, Elfadil 0, Abdulrazaq G, Osman S, Khan $M M$, Ahmed A, et al. Favorable Outcome of COVID-19 Pneumonia in a Kidney Transplant Recipient Treated with Tocilizumab. Case Rep Infect Dis. 2020;2020:8830626.

68. Trujillo $H$, Caravaca-Fontán $F$, Sevillano A, Gutiérrez $\mathrm{E}$, Fernández-Ruiz $\mathrm{M}$, López-Medrano $\mathrm{F}$, et al. Tocilizumab use in Kidney Transplant Patients with $\mathrm{CO}$ VID-19. Clin Transplant. 2020;34(11):e14072.

69. Pérez-Sáez MJ, Blasco $M$, Redondo-Pachón $D$, Ventura-Agújar $P$, Bada-Bosch $T$, Pérez-Flores $\mathrm{I}$, et al. Use of tocilizumab in kidney transplant recipients with COVID-19. Am J Transplant. 2020;20(11):3182-90.

70. Nishimoto N, Terao K, Mima T, Nakahara H, Takagi $\mathrm{N}$, Kakehi T. Mechanisms and pathologic significances in increase in serum interleukin-6 (IL-6) and soluble IL-6 receptor after administration of an anti-IL-6 receptor antibody, tocilizumab, in patients with rheumatoid arthritis and Castleman disease. Blood. 2008;112(10):3959-64.

71. Ringer M, Azmy V, Kaman K, Tang D, Cheung H, Azar $M$, et al. A retrospective matched cohort single-center study evaluating outcomes of $\mathrm{CO}$ -
VID-19 and the impact of immunomodulation on COVID-19-related cytokine release syndrome in solid organ transplant recipients. Transpl Infect Dis. 2021;23(2):e13556.

72. Pereira MR, Aversa MM, Farr MA, Miko FA, Aaron JG, Mohan S, et al. Tocilizumab for severe COVID-19 in solid organ transplant recipients: a matched cohort study. Am J Transplant. 2020;20(11):3198-205.

73. Bodro M, Cofan F, Rios J, Herrera S, Linares L, Mar$\cos M A$, et al. Use of Anti-Cytokine Therapy in Kidney Transplant Recipients with COVID-19. J Clin Med. 2021;10(8).

74. Pulakurthi YS, Pederson JM, Saravu K, Gupta N, Balasubramanian $\mathrm{P}$, Kamrowski S, et al. Corticosteroid therapy for COVID-19: A systematic review and meta-analysis of randomized controlled trials. Medicine (Baltimore). 2021;100(20):e25719.

75. Karruli A, Spiezia S, Boccia F, Gagliardi M, Patauner F, Salemme A, et al. Effect of immunosuppression maintenance in solid organ transplant recipients with COVID-19: Systematic review and meta-analysis. Transpl Infect Dis. 2021:e13595.

76. Thompson MG, Burgess JL, Naleway AL, Tyner $H$, Yoon SK, Meece J, et al. Prevention and Attenuation of COVID-19 with the BNT162b2 and mRNA-1273 Vaccines. N Engl J Med. 2021.

77. Baden LR, El Sahly HM, Essink B, Kotloff K, Frey $S$, Novak $R$, et al. Efficacy and Safety of the mRNA-1273 SARS-CoV-2 Vaccine. N Engl J Med. 2021;384(5):403-16.

78. Cucchiari D, Egri N, Bodro M, Herrera S, Del Risco-Zevallos J, Casals-Urquiza J, et al. Cellular and humoral response after mRNA-1273 SARS-CoV-2 vaccine in kidney transplant recipients. Am J Transplant. 2021;21(8):2727-39.

79. Boyarsky BJ, Werbel WA, Avery RK, Tobian AAR, Massie $A B$, Segev DL, et al. Antibody Response to 2-Dose SARS-CoV-2 mRNA Vaccine Series in Solid Organ Transplant Recipients. JAMA. 2021;325(21):2204-6.

80. Malinis M, Cohen E, Azar MM. Effectiveness of SARS-CoV-2 vaccination in fully vaccinated solid organ transplant recipients. Am J Transplant. 2021;21(8):2916-8. 
81. Ali NM, Alnazari N, Mehta SA, Boyarsky B, Avery RK, Segev DL, et al. Development of COVID-19 Infection in Transplant Recipients After SARS-CoV-2 Vaccination. Transplantation. 2021;105(9):e104-6.

82. Rozen-Zvi B, Yahav D, Agur T, Zingerman B, Ben-Zvi $\mathrm{H}$, Atamna $\mathrm{A}$, et al. Antibody response to SARS-CoV-2 mRNA vaccine among kidney transplant recipients: a prospective cohort study. Clin Microbiol Infect. 2021;27(8):1173.e1-1173.e4.

83. Baluch A, Humar A, Eurich D, Egli A, Liacini A, Hoschler $\mathrm{K}$, et al. Randomized controlled trial of high-dose intradermal versus standard-dose intramuscular influenza vaccine in organ transplant recipients. Am J Transplant. 2013;13(4):1026-33.

84. Grupper A, Rabinowich L, Schwartz D, Schwartz IF, Ben-Yehoyada M, Sashar M, et al. Reduced humoral response to mRNA SARS-CoV-2 BNT162b2 vaccine in kidney transplant recipients without prior exposure to the virus. Am J Transplant. 2021;21(8):2719-26.

85. Danthu C, Hantz S, Dahlem A, Duval M, Ba B, Guibbert $M$, et al. Humoral Response after SARS-CoV-2 mRNA Vaccine in a Cohort of Hemodialysis Patients and Kidney Transplant Recipients. J Am Soc Nephrol. 2021;32(9):2153-8.

86. Chavarot N, Ouedrani A, Marion O, Leruez-Ville M, Vilain E, Baaziz M, et al. Poor Anti-SARS-CoV-2 Humoral and T-cell Responses After 2 Injections of mRNA Vaccine in Kidney Transplant Recipients Treated with Belatacept. Transplantation. 2021;105(9):e94-5.

87. Ou MT, Boyarsky BJ, Chiang TPY, Bae S, Werbel WA, Avery RK, et al. Immunogenicity and Reactogenicity After SARS-CoV-2 mRNA Vaccination in Kidney Transplant Recipients Taking Belatacept. Transplantation. 2021;105(9):2119-23.

88. Benotmane I, Gautier-Vargas $G$, Gallais $F$, Gantner $P$, Cognard N, Olagne J, et al. Strong antibody response after a first dose of a SARS-CoV-2 mRNA-based vaccine in kidney transplant recipients with a previous history of COVID-19. Am J Transplant. 2021. [en prensa]. D0I: https://doi.org/10.1111/ajt.16764.

89. Ou MT, Boyarsky BJ, Motter JD, Greenberg RS, Teles AT, Ruddy JA, et al. Safety and Reactogenicity of 2 Doses of SARS-CoV-2 Vaccination in Solid Organ Transplant Recipients. Transplantation. 2021. [en prensa]. D0I: https://doi.org/ 10.1097/ TP.0000000000003780.

90. Kamar N, Abravanel F, Marion 0, Couat C, Izopet J, Del Bello A. Three Doses of an mRNA COVID-19 Vaccine in Solid-Organ Transplant Recipients. N Engl J Med. 2021;385:661-2.

91. Werbel WA, Boyarsky BJ, Ou MT, Massie AB, Tobian AAR, Garonzik-Wang J, et al. Safety and Immunogenicity of a Third Dose of SARS-CoV-2 Vaccine in Solid Organ Transplant Recipients: A Case Series. Ann Intern Med. 2021;174:1330-2.

92. Mamode N, Ahmed Z, Jones G, Banga N, Motallebzadeh $\mathrm{R}$, Tolley $\mathrm{H}$, et al. Mortality Rates in Transplant Recipients and Transplantation Candidates in a High-prevalence COVID-19 Environment. Transplantation. 2021;105(1):212-5.

93. Al Otaibi TM, Gheith $O A$, Abuelmagd MM, Adel M, Alqallaf AK, Elserwy NA et al. Better outcome of COVID-19 positive kidney transplant recipients during the unremitting stage with optimized anticoagulation and immunosuppression. Clin Transplant. 2021;35(6):e14297.

94. Alshaqaq A, Al Abadi A, Altheaby A, Bukhari M, Alnasrallah $B$, Alamoudi $A$, et al. Coronavirus Disease 2019 and Kidney Transplantation in Saudi Arabia: Outcomes and Future Opportunities. Ann Transplant. 2021;26:e931832.

95. Caillard S, Chavarot N, Francois H, Matignon M, Snanoudj $\mathrm{R}$, Tourret J, et al. Clinical utility of biochemical markers for the prediction of COVID-19-related mortality in kidney transplant recipients. Kidney Int Rep. 2021. [en prensa]. doi: 10.1016/j.ekir.2021.06.034.

Este artículo se distribuye bajo una Licencia Creative Commons Atribución-NoComercial 4.0 Internacional. https://creativecommons.org/licenses/by-nc/4.0/

Open Access (c) (1) \& 